\title{
Convex computation of the maximum controlled invariant set for discrete-time polynomial control systems ${ }^{\star}$
}

\author{
Milan Korda ${ }^{1}$, Didier Henrion ${ }^{2,3,4}$, Colin N. Jones ${ }^{1}$
}

\begin{abstract}
We characterize the maximum controlled invariant (MCI) set for discrete-time systems as the solution of an infinite-dimensional linear programming problem. In the case of systems with polynomial dynamics and semialgebraic state and control constraints, we describe a hierarchy of finitedimensional linear matrix inequality relaxations of this problem that provides outer approximations with guaranteed set-wise convergence to the MCI set. The approach is compact and readily applicable in the sense that the approximations are the outcome of a single semidefinite program with no additional input apart from the problem description.
\end{abstract}

\section{INTRODUCTION}

Given a discrete-time controlled dynamical system described, its maximum controlled invariant (MCI) set is the set of all initial states that can be kept within a given constraint set ad infinitum using admissible control inputs. This set goes by many other names in the literature, e.g., viability kernel in viability theory [4], or $(A, B)$-invariant set in the linear case [13].

Set invariance is an ubiquitous and essential concept in dynamical systems theory, as far as both analysis and control synthesis is concerned. In particular, by its very definition, the MCI set determines fundamental limitations of a given control system with respect to constraint satisfaction. In addition, there is a very tight link between invariant sets and (control) Lyapunov functions. Indeed, sub-level sets of a Lyapunov function give rise to invariant sets. Conversely, at least in the linear case, any controlled invariant set gives rise to a control Lyapunov function, and therefore these sets can be readily used to design stabilizing control laws; see, e.g., [8] for a general treatment and, e.g., [17], [25] for applications in model predictive control design.

The problem of (maximum) controlled invariant set computation for discrete-time systems has been a topic of active research for more than four decades. The central tool in this effort has been the contractive algorithm of [6] and its expansive counterpart [18]. For an exhaustive survey and historical remarks see the survey [8] and the book [12].

Both algorithms, although conceptually applicable to any nonlinear system, have been predominantly applied in a linear setting where they boil down to a sequence of linear programs and polyhedral projections. Finite termination of this sequence is a subtle problem and sharp results are available only in the uncontrolled setting where no projections are required [16]; for discussion of finite-termination in

\footnotetext{
*An extended version of the paper treating also continuous-time is available from arXiv:1303.6469.

${ }^{1}$ Laboratoire d'Automatique, École Polytechnique Fédérale de Lausanne, Station 9, CH-1015, Lausanne, Switzerland. \{milan.korda, colin.jones\}@epfl.ch

${ }^{2}$ CNRS; LAAS; 7 avenue du colonel Roche, F-31400 Toulouse; France. henrionelaas. fr

${ }^{3}$ Université de Toulouse; LAAS; F-31400 Toulouse; France.

${ }^{4}$ Faculty of Electrical Engineering, Czech Technical University in Prague, Technická 2, CZ-16626 Prague, Czech Republic.
}

the controlled case see [39]. The contractive and expansive algorithms were combined in [17] to design an algorithm terminating in a finite number of iterations and outputting an $\epsilon$-accurate inner approximation of the MCI set (with the accuracy measured by the Hausdorff distance). Another line of research, culminating in [37], exploits the linearity of the system dynamics in a more systematic way and approximates the maximum (or minimum) robust controlled invariant set by the Minkowski sum of a parametrized family of sets. Very recently, in continuous time, [29] developed a parallel algorithm for ellipsoidal approximations of the robust $\mathrm{MCI}$ set scalable to very high dimensions. Computation of lowcomplexity polyhedral controlled invariant sets was investigated in [10] and [11].

In the nonlinear case, a common practice is to exploit the tight connection between invariance and Lyapunov functions and seek invariant sets as sub-level sets of a (control) Lyapunov function; see [14] and references therein discussing the related problem of region of attraction computation in continuous time. This, however, typically leads to nonconvex bilinear optimization problems which are notoriously hard to solve. Therefore one often has to resort to ad-hoc analysis of the specific system at hand, which is typically tractable only in small dimensions; see [40], [41] for concrete examples in continuous time. Related in spirit is the localization technique of [24] for discrete-time autonomous systems, also requiring considerable effort in analysing the system. Recently, a general approach using finite-dimensional linear programming (LP) was used in [5] to design a controller ensuring invariance of a given candidate polyhedral set.

Building upon our previous work [19] on the computation of the region of attraction (ROA) for polynomial control systems, in this paper we characterize the maximum controlled invariant (MCI) set for discrete-time polynomial systems as the solution to an infinite-dimensional LP problem in the cone of nonnegative measures. The dual of this problem is an infinite-dimensional LP in the space of continuous functions. Finite-dimensional relaxations of the primal LP and finitedimensional approximations of the dual LP turn out to be semidefinite programs (SDPs) also related by duality. The primal relaxations lead to a truncated moment problem while the dual approximations to a sum-of-squares (SOS) problem. Super-level sets of one of the polynomials appearing in the dual SOS problem then provide outer approximations to the MCI set with guaranteed convergence as the degree of the polynomial tends to infinity.

The main mathematical tool we use are the so-called occupation measures which allow us to study the time evolution of the whole ensemble of initial conditions (described by a measure) rather than studying trajectories associated to each initial condition separately. The use of measures to study dynamical systems has a very long tradition: see [38] for 
probably the first systematic treatment ${ }^{1}$ in continuous time; for purely discrete-time treatment see [22, Chapter 6]. To the best of the authors' knowledge our paper is the first one to use occupation measures for MCI set (approximate) computation. The MCI set was previously characterized using occupation measures in [15], but there the characterization is rather indirect and not straightforwardly amenable to computation. Apart from the authors' work [19], the related problem of region of attraction computation was tackled using measures in [45]. There, however, a very different approach was taken, not using occupation measures but rather analyzing convergence via discretization of the statespace and propagating the initial distribution by means of a discretized transfer operator. Here, instead, we employ the (discounted) occupation measure which captures the behaviour of the trajectories emanating from the initial distribution over the infinite time horizon. As a result, our approach requires no discretization and, contrary to [45], provides true guarantees (not in an "almost-everywhere" or "coarse" sense) and, more importantly, is applicable in a controlled setting.

Similar in spirit to our approach, from the dual viewpoint of optimization over functions, are the HamiltonJacobi approaches (e.g., [33], [34]) although, importantly, our approach does not require state-space discretization and comes with convergence guarantees.

What can be considered a drawback of our approach is the fact that the approximations to the MCI set we obtain are from the outside and therefore not invariant. However, accurate outer approximations provide important information as to the performance limitations of the control system and are of practical interest, e.g., in collision avoidance. Therefore we believe that our work bears both theoretical and practical value, and naturally complements existing innerapproximation techniques.

The paper is organised as follows. The problem to be solved is described in Section II. Occupation measures are introduced in Section III. The infinite-dimensional primal and dual LPs are described in Sections IV and V, respectively. The finite-dimensional relaxations with convergence results are presented in Section VI. Numerical examples are in Section VII. A reader interested only in the semialgebraic outer approximations of the MCI set can consult directly the infinite-dimensional dual LP (5) and its finite-dimensional approximation (7).

\section{PRoblem STATEMENT}

Consider the discrete-time control system

$$
x_{t+1}=f\left(x_{t}, u_{t}\right), \quad x_{t} \in X, \quad u_{t} \in U, \quad t \in\{0,1, \ldots\}
$$

with a given polynomial vector field $f$ with entries $f_{i} \in$ $\mathbb{R}[x, u], i=1, \ldots, n$, and given compact basic semialgebraic state and input constraints

$$
\begin{gathered}
x_{t} \in X:=\left\{x \in \mathbb{R}^{n}: g_{X_{i}}(x) \geq 0, i=1,2, \ldots, n_{X}\right\}, \\
u_{t} \in U:=\left\{u \in \mathbb{R}^{m}: g_{U_{i}}(u) \geq 0, i=1,2, \ldots, n_{U}\right\}
\end{gathered}
$$

with $g_{X_{i}} \in \mathbb{R}[x], g_{U_{i}} \in \mathbb{R}[u]$.

\footnotetext{
${ }^{1}$ In [38], J. E. Rubio used Young measures [44] rather than occupation measures, but the basic idea of "linearizing" a nonlinear problem by going into an infinite-dimensional space of measures is the same.
}

The maximum controlled invariant (MCI) set is defined as

$$
\begin{gathered}
X_{I}:=\left\{x_{0} \in X: \exists\left(\left\{x_{t}\right\}_{t=1}^{\infty},\left\{u_{t}\right\}_{t=1}^{\infty}\right) \text { s.t. } x_{t+1}=f\left(x_{t}, u_{t}\right),\right. \\
\left.u_{t} \in U, x_{t} \in X, \forall t \in\{0,1, \ldots\}\right\} .
\end{gathered}
$$

A control sequence $\left\{u_{t}\right\}_{t=0}^{\infty}$ is called admissible if $u_{t} \in U$ for all $t \in\{0,1, \ldots\}$.

In words, the MCI set is the set of all initial states which can be kept inside the constraint set $X$ ad infinitum using admissible control inputs.

\section{OCCUPATION MEASURES}

In this section we introduce the concept of occupation measures which is the centrepiece of our approach.

For the reader's ease, let us briefly survey the measuretheoretic notation used in this paper. We work with signed Borel measures on a Euclidean space, understood as countably additive maps from the Borel sets (the smallest sigmaalgebra containing open sets) to the real numbers. The vector space of all signed Borel measures with its support contained in a Borel set $K$ is denoted by $M(K)$. The support (i.e., the smallest closed set whose complement has a zero measure) of a measure $\nu$ is denoted by $\operatorname{spt} \nu$. The space of continuous functions on $K$ is denoted by $C(K)$. The indicator function of a set $K$ (i.e., a function equal to one on $K$ and zero otherwise) is denoted by $I_{K}(\cdot)$. The symbol $\lambda$ denotes the $n$-dimensional Lebesgue measure (i.e., the standard $n$-dimensional volume). The integral of a function $v$ w.r.t a measure $\nu$ over a set $K$ is denoted by $\int_{K} v(x) d \nu(x)$. Sometimes for conciseness we use the shorter notation $\int v d \mu$ omitting the integration variable and also the set over which we integrate if they are obvious from the context. Finally, the ring of polynomials in a variable $x$ is denoted by $\mathbb{R}[x]$.

Given a discount factor $\alpha \in(0,1)$, an initial condition $x_{0}$ and an admissible control sequence $\left\{u_{t \mid x_{0}}\right\}_{t=0}^{\infty}$ such that the associated state sequence $\left\{x_{t \mid x_{0}}\right\}_{t=0}^{\infty}$ remains in $X$ for all times, we define the discounted occupation measure $\mu(\cdot \mid$ $\left.x_{0}\right) \in M(X \times U)$ as

$$
\mu\left(A \times B \mid x_{0}\right):=\sum_{t=0}^{\infty} \alpha^{t} I_{A \times B}\left(x_{t \mid x_{0}}, u_{t \mid x_{0}}\right)
$$

for all sets $A \subset X$ and $B \subset U$.

In words, the discounted occupation measure measures the (discounted) number of visits of the state-control pair trajectory $\left(x\left(\cdot \mid x_{0}\right), \nu\left(\cdot \mid x_{0}\right)\right)$ to subsets of $X \times U$. The discounting in the definition of the occupation measure ensures that $\mu\left(A \times B \mid x_{0}\right)$ is always finite; in fact we have $\mu\left(X \times U \mid x_{0}\right)=(1-\alpha)^{-1}$.

Now suppose that the initial condition is not a single point but an initial measure $\mu_{0} \in M(X)$ and an admissible control sequence is associated to each initial condition from the support of $\mu_{0}$ in such a way that the corresponding state sequence remains in $X$. Then we define the average discounted occupation measure $\mu \in M(X \times U)$ as

$$
\mu(A \times B):=\int_{X} \mu\left(A \times B \mid x_{0}\right) d \mu_{0}\left(x_{0}\right) .
$$

\footnotetext{
${ }^{2}$ The initial measure $\mu_{0}$ can be thought of as the probability distribution of the initial state, although we do not require the mass of $\mu_{0}$ to be normalized to one.
} 
The average discounted occupation measure measures the discounted average number of visits in subsets of $X \times U$ of trajectories starting from the initial distribution $\mu_{0}$.

Now we derive an equation linking the measures $\mu_{0}$ and $\mu$. This equation will play a key role in subsequent development and in a sense replaces the dynamics equation (1). To derive this equation fix an initial condition $x_{0} \in X$ and a control sequence $\left\{u_{t \mid x_{0}}\right\}_{t=0}^{\infty}$ such that the associated state sequence $\left\{x_{t \mid x_{0}}\right\}_{t=0}^{\infty}$ stays in $X$. Then for any $v \in C(X)$ we have

$$
\begin{aligned}
\int_{X \times U} v(x) d \mu\left(x, u \mid x_{0}\right) \\
=\sum_{t=0}^{\infty} \alpha^{t} v\left(x_{t \mid x_{0}}\right)=v\left(x_{0 \mid x_{0}}\right)+\alpha \sum_{t=0}^{\infty} \alpha^{t} v\left(x_{t+1 \mid x_{0}}\right) \\
=v\left(x_{0 \mid x_{0}}\right)+\alpha \sum_{t=0}^{\infty} \alpha^{t} v\left(f\left(x_{t \mid x_{0}}, u_{t \mid x_{0}}\right)\right) \\
=v\left(x_{0 \mid x_{0}}\right)+\alpha \int_{X \times U} v(f(x, u)) d \mu\left(x, u \mid x_{0}\right) .
\end{aligned}
$$

Integrating w.r.t. $\mu_{0}$ we arrive at the sought equation

$$
\begin{array}{r}
\int_{X \times U} v(x) d \mu(x, u)=\int_{X} v(x) d \mu_{0}(x)+\alpha \int_{X \times U} v(f(x, u)) d \mu(x, u) \\
\forall v \in C(X) .
\end{array}
$$

Note that this is an infinite-dimensional linear equation in variables $\left(\mu_{0}, \mu\right)$.

The following crucial Lemma establishes the connection between the support of any initial measure $\mu_{0}$ solving (3) and the MCI set $X_{I}$.

Lemma 1. For any pair of measures $\left(\mu_{0}, \mu\right)$ satisfying equation (3) with spt $\mu_{0} \subset X$ and spt $\mu \subset U \times X$ we have spt $\mu_{0} \subset X_{I}$.

Proof. A detailed proof is in the Appendix A of the extended version of the paper available from arXiv:1303.6469.

\section{PRIMAL LP}

In this section we show how the MCI set computation problem can be cast as an infinite-dimensional LP problem in the cone of nonnegative measures. As in [19], the basic idea is to maximize the mass of the initial measure $\mu_{0}$ subject to the constraint that it be dominated by the Lebesgue measure, that is, $\mu_{0} \leq \lambda$. System dynamics is captured by the equations (3), and state and input constraints are expressed through constraints on the supports of the initial and occupation measure. The constraint that $\mu_{0} \leq \lambda$ can be equivalently rewritten as $\mu_{0}+\hat{\mu}_{0}=\lambda$ for some nonnegative slack measure $\hat{\mu}_{0} \in M(X)$. This constraint is in turn equivalent to $\int_{X} w(x) d \mu_{0}(x)+\int_{X} w(x) d \hat{\mu}_{0}(x)=\int_{X} w(x) d \lambda(x)$ for all $w \in C(X)$. These considerations lead to the following primal LPs.

The primal LP reads

$$
\begin{array}{rlrl}
p^{*}= & \sup \mu_{0}(X) & \\
\text { s.t. } & \int v d \mu=\int v d \mu_{0}+\alpha \int v \circ f d \mu & & \forall v \in C(X) \\
& \int w d \mu_{0}+\int w d \hat{\mu}_{0}=\int w d \lambda & & \forall w \in C(X) \\
& \mu \geq 0, \mu_{0} \geq 0, \hat{\mu}_{0} \geq 0 & \\
& \operatorname{spt} \mu \subset X \times U, \text { spt } \mu_{0} \subset X, \text { spt } \hat{\mu}_{0} \subset X,
\end{array}
$$

where the infimum is over the vector of measures $\left(\mu, \mu_{0}, \hat{\mu}_{0}\right) \in M(X \times U) \times M(X) \times M(X)$.

This is an infinite-dimensional LP in the cone of nonnegative Borel measures. The following Lemma, which is our main theoretical result, relates an optimal solution of this LP to the MCI set $X_{I}$.

Theorem 1. The optimal value of LP problem (4) is equal to the volume of the MCI set $X_{I}$, that is, $p^{*}=\lambda\left(X_{I}\right)$. Moreover, the supremum is attained by the restriction of the Lebesgue measure to the MCI set $X_{I}$.

Proof. The proof follows from Lemma 1 by the same arguments as Theorem 1 in [19]. By definition of the MCI set $X_{I}$, for any initial condition $x_{0} \in X_{I}$ there exists an admissible control sequence such that the associated state sequence remains in $X$. Therefore for any initial measure $\mu_{0} \leq \lambda$ with spt $\mu_{0} \subset X_{I}$ there exist a discounted occupation measure $\mu$ with spt $\mu \subset X \times U$ and a slack measure $\hat{\mu}_{0}$ with spt $\hat{\mu}_{0} \subset X$ such that the constraints of problem (4) are satisfied. One such measure $\mu_{0}$ is the restriction of the Lebesgue measure to $X_{I}$, and therefore $p^{*} \geq \lambda\left(X_{I}\right)$. The fact $p^{*} \leq \lambda\left(X_{I}\right)$ follows from Lemma 1 .

\section{DUAL LP}

In this section we derive an LP dual to LP (4). Since the primal LP is on the space of measures, the dual LP will be on the space of continuous functions. Super-level sets of feasible solutions to this dual LP then provide outer approximations to the MCI set. The dual can be derived by standard infinite-dimensional LP duality theory; see [19] for a derivation in a similar setting or [3] for a general theory of infinite-dimensional linear programming.

The dual LP reads

$$
\begin{array}{rlrl}
d^{*}=\inf & \int_{X} w(x) d \lambda(x) & \\
\text { s.t. } & \alpha v(f(x, u)) \leq v(x), & & \forall(x, u) \in X \times U \\
& w(x) \geq v(x)+1, & & \forall x \in X \\
& w(x) \geq 0, & & \forall x \in X,
\end{array}
$$

where the infimum is over the pair of functions $(v, w) \in$ $C(X) \times C(X)$.

The following key observation shows that the unit superlevel set of any function $w$ feasible in (5) provides an outerapproximation to $X_{I}$.

Lemma 2. Any feasible solution to problem (5) satisfies $w \geq$ 1 on $X_{I}$.

Proof. Given any $x_{0} \in X_{I}$ there exists a sequence $\left\{u_{t}\right\}_{t=0}^{\infty}$, $u_{t} \in U$, such that $x_{t} \in X$ for all $t$. The first constraint of problem (5) is equivalent to $\alpha v\left(x_{t+1}\right) \leq v\left(x_{t}\right), t \in$ $\{0,1, \ldots\}$. By iterating this inequality we get

$$
v\left(x_{0}\right) \geq \alpha^{t} v\left(x_{t}\right) \rightarrow 0 \quad \text { as } \quad t \rightarrow \infty
$$

since $x_{t} \in X$ and $X$ is bounded. Therefore $v\left(x_{0}\right) \geq 0$ and $w\left(x_{0}\right) \geq 1$ for all $x_{0} \in X_{I}$.

The following theorem is instrumental in proving the convergence results of Section VI.

Theorem 2. There is no duality gap between primal LP problems (4) on measures and dual LP problem (5) on functions in the sense that $p^{*}=d^{*}$. 
Proof. Follows by the same arguments as Theorem 2 in [19].

\section{LMI RELAXATIONS}

In this section we present finite-dimensional relaxations of the infinite-dimensional LPs. The relaxations of the primal LP lead to a truncated moment problem which translates to a semidefinite program (SDP) that can be solved by freely available software, e.g., SeDuMi [35] or SDPA [42]. Dual to the primal SDP relaxation is a sum-of-squares (SOS) problem that again translates to an SDP problem. The following discussion closely follows the one in [26].

We only highlight the main ideas behind the derivation of the finite-dimensional relaxations. The reader is referred to [19, Section 5] or to the comprehensive reference [30] for details. First, since the supports of all measures feasible in (4) are compact, these measures are uniquely determined by their moments, i.e., by integrals of all monomials (which is a sequence of real numbers when indexed in, e.g., the canonical monomial basis). Therefore, it suffices to restrict the test functions $w(x)$ and $v(x)$ in (4) to all monomials, reducing the linear equality constraints on measures $\mu_{0}, \mu$ and $\hat{\mu}_{0}$ of (4) to linear equality constraints on their moments. Next, by the Putinar Positivstellensatz (see [30], [36]), the constraint that the support of a measure is included in a given compact basic semialgebraic set is equivalent to the feasibility of an infinite sequence of LMIs involving the so-called moment and localizing matrices, which are linear in the coefficients of the moment sequence. By truncating the moment sequence and taking only the moments corresponding to monomials of total degree less than or equal to $2 k$ we obtain a necessary condition for this truncated moment sequence to be the first part of a moment sequence corresponding to a measure with the desired support.

In what follows, $\mathbb{R}_{k}[\cdot]$ denotes the vector space of real multivariate polynomials of total degree less than or equal to $k$. Furthermore, throughout the rest of this section we make the following standard standing assumption:

Assumption 1. One of the polynomials modeling the sets $X$ resp. $U$ is equal to $g_{X_{i}}(x)=R_{X}^{2}-\|x\|_{2}^{2}$ resp. $g_{U_{i}}(u)=$ $R_{U}^{2}-\|u\|_{2}^{2}$ with $R_{X}, R_{U}$ sufficiently large constants.

This assumption is completely without loss of generality since redundant ball constraints can be always added to the description of the compact sets $X$ and $U$.

The primal relaxation of order $k$ reads

$$
\begin{aligned}
& p_{k}^{*}=\max \left(y_{0}\right)_{0} \\
& \text { s.t. } \quad A_{k}\left(y, y_{0}, \hat{y}_{0}\right)=b_{k} \\
& M_{k}(y) \succeq 0, \quad M_{k-d_{X i}}\left(g_{X i}, y\right) \succeq 0, \quad i=1, \ldots, n_{X} \\
& M_{k-d_{U i}}\left(g_{U_{i}}, y\right) \succeq 0, \quad i=1, \ldots, n_{U} \\
& M_{k}\left(y_{0}\right) \succeq 0, \quad M_{k-d_{X} i}\left(g_{X}, y_{0}\right) \succeq 0, \quad i=1, \ldots, n_{X} \\
& M_{k}\left(\hat{y}_{0}\right) \succeq 0, \quad M_{k-d_{X_{i}}}\left(g_{X_{i}}, \hat{y}_{0}\right) \succeq 0, \quad i=1, \ldots, n_{X},
\end{aligned}
$$

where the notation $\succeq 0$ stands for positive semidefinite and the minimum is over moment sequences $\left(y, y_{0}, \hat{y}_{0}\right)$ truncated to degree $2 k$ corresponding to measures $\mu, \mu_{0}$ and $\hat{\mu}_{0}$ in (4). The linear equality constraint captures the two linear equality constraints of (4) with $v(t, x) \in \mathbb{R}_{2 k}[t, x]$ and $w(x) \in \mathbb{R}_{2 k}[x]$ being monomials of total degree less than or equal to $2 k$. The matrices $M_{k}(\cdot)$ are the moment and localizing matrices, following the notation of [30] or [19]. In problem (6), a linear objective is minimized subject to linear equality constraints and LMI constraints; therefore problem (6) is a semidefinite program (SDP).

The dual relaxation of order $k$ reads

$$
\begin{aligned}
d_{k}^{*}=\inf \quad \boldsymbol{w}^{\prime} l \\
\text { s.t. } \quad v-\alpha \cdot v \circ f=q_{0}+\sum_{i=1}^{n_{X}} q_{i} g_{X}+\sum_{i=1}^{n_{U}} r_{i} g_{U} \\
\\
w-v-1=p_{0}+\sum_{i=1}^{n_{X}} p_{i} g_{X} i \\
w=s_{0}+\sum_{i=1}^{n_{X}} s_{i} g_{X},
\end{aligned}
$$

where $l$ is the vector of Lebesgue moments over $X$ indexed in the same basis in which the polynomial $w(x)$ with coefficient vector $\boldsymbol{w}$ is expressed. The minimum is over polynomials $v(x) \in \mathbb{R}_{2 k}[x]$ and $w \in \mathbb{R}_{2 k}[x]$, and polynomial sum-ofsquares $q_{i}(x, u), p_{i}(x), s_{i}(x), i=1, \ldots, n_{X}$ and $r_{i}(x, u)$, $i=1, \ldots, n_{U}$, of appropriate degrees. In problem (7), a linear objective function is minimized subject to sum-ofsquares (SOS) constraints; therefore problem (7) is an SOS problem which can be readily cast as an SDP (see, e.g., [30]).

\section{A. Convergence results}

In this section we state several convergence results of the finite dimensional relaxations. The proofs of the results follow exactly the same reasoning as analogous results on region of attraction approximations in [19, Section 6] and are omitted for space reasons.

Theorem 3. There is no duality gap between the primal LMI problem (6) and the dual LMI problem (7), i.e., $p_{k}^{*}=d_{k}^{*}$.

The next theorem shows functional convergence from above to the indicator function of the MCI set.

Theorem 4. Let $w_{k} \in \mathbb{R}_{2 k}[x]$ denote the $w$-component of a solution to the dual LMI problem (7) and let $\bar{w}_{k}(x)=$ $\min _{i \leq k} w_{i}(x)$. Then $w_{k}$ converges from above to $I_{X_{I}}$ in $L^{1}$ norm and $\bar{w}_{k}$ converges from above to $I_{X_{I}}$ in $L^{1}$ norm and almost uniformly.

The following immediate corollary shows the convergence of the optimal values of the relaxations to the optimal values of the infinite-dimensional LPs.

Corollary 1. The sequence of infima of LMI problems (7) converges monotonically from above to the supremum of the LP problem (5), i.e., $d^{*} \leq d_{k+1}^{*} \leq d_{k}^{*}$ and $\lim _{k \rightarrow \infty} d_{k}^{*}=$ $d^{*}$. Similarly, the sequence of maxima of LMI problems (6) converges monotonically from above to the maximum of the LP problem (4), i.e., $p^{*} \leq p_{k+1}^{*} \leq p_{k}^{*}$ and $\lim _{k \rightarrow \infty} p_{k}^{*}=p^{*}$.

Our last theorem shows a set-wise convergence of the outer-approximations to the MCI set.

Theorem 5. Let $w_{k} \in \mathbb{R}_{2 k}[x]$ denote the $w$-component of a solution to the dual LMI problem (7) and let $X_{I k}:=\{x \in$ $\left.\mathbb{R}^{n}: w_{k}(x) \geq 1\right\}$. Then $X_{I} \subset X_{I k}$,

$$
\lim _{k \rightarrow \infty} \lambda\left(X_{I k} \backslash X_{I}\right)=0 \text { and } \lambda\left(\cap_{k=1}^{\infty} X_{I k} \backslash X_{I}\right)=0 .
$$

\section{NUMERICAL EXAMPLES}

In this section we present numerical examples that illustrate our results. The primal LPs on measures were modeled using Gloptipoly 3 [20] and the primal SDP relaxations were solved using SeDuMi [35], which automatically returns also the dual solutions providing the outer approximations. 
1) Julia sets: Consider over $z \in \mathbb{C}$, or equivalently over $x \in \mathbb{R}^{2}$ with $z:=x_{1}+i x_{2}$, the quadratic recurrence

$$
z^{+}=z^{2}+c
$$

with $c \in \mathbb{C}$ a given complex number and $i$ the imaginary unit. The filled Julia set is the set of all initial conditions of the above recurrence for which the trajectories remain bounded. The shape of the Julia set depends strongly on the parameter $c$. If $c$ lies inside the Mandelbrot set, then the Julia set is connected; otherwise the set is disconnected. In both cases the boundary of the set has a very complicated (in fact fractal) structure. Here we shall compute outer approximations of the filled Julia set intersected with the unit ball. To this end we set $X=\left\{x \in \mathbb{R}^{2}:\|x\| \leq 1\right\}$. Figure 1 shows outer approximations of degree 12 for parameter values $c=-0.7+i 0.2$ (inside the Mandelbrot set) and $c=-0.9+i 0.2$ (outside the Mandelbrot set). The "true" filled Julia set was (approximately) obtained by by randomly sampling initial conditions within the unit ball and iterating the recurrence for one hundred steps. Taking higher degree of the approximating polynomials does not give significant improvements due to our choice of the monomial basis to represent polynomials. An alternative basis (e.g. Chebyshev polynomials - see the related discussions in [21] and [19]) would allow us to improve further the outer estimates and better capture the fine fractal structure of the filled Julia set's boundary.

2) Hénon map: Consider the modified controlled Hénon map [31]

$$
\begin{aligned}
& x_{1}^{+}=0.44-0.1 x_{3}-4 x_{2}^{2}+0.5 u, \\
& x_{2}^{+}=x_{1}-4 x_{1} x_{2}, \\
& x_{3}^{+}=x_{2},
\end{aligned}
$$

with $=X=[-1,1]^{3}$ and $U=\left[-u_{\max }, u_{\max }\right]$. We investigate two cases: uncontrolled (i.e., $u_{\max }=0$ ) and controlled with $u_{\max }=1$. Figure 2 shows outer approximations to the MCI set of degree eight for both settings and the "true" MCI set in the uncontrolled setting (approximately) obtained by random sampling of initial conditions inside the constraint set $X$. The outer approximations suggest that, as expected, allowing for control leads to a larger MCI set.

\section{CONCLUSION}

We derived an infinite-dimensional convex characterization of the maximum controlled invariant (MCI) set, finitedimensional approximations of (the dual of) which provide a converging sequence of semialgebraic outer-approximations to this set. The outer-approximations are the outcome of a single semidefinite program (SDP) with no additional data required besides the problem description. Therefore the approach is readily applicable using freely available modeling tools such Gloptipoly 3 [20] or YALMIP [32] with no hand-tuning involved.

The cost to pay for this comfort is the relatively unfavourable scalability of the semidefinite programs solved - the number of variables grows as $O\left((n+m)^{d}\right)$, where $n$ and $m$ are the state and control dimensions and $d$ is the degree of the approximating polynomial. Therefore, in order for this approach to scale to medium dimensions (say, more than $m+n=6$ ) one either has to tradeoff accuracy by taking small $d$ or go beyond the standard freely available solvers

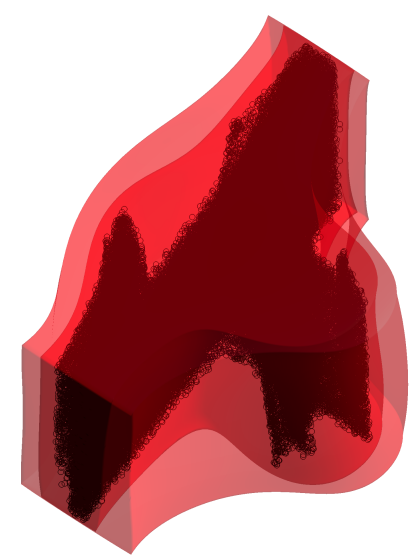

Fig. 2. Hénon map - polynomial outer approximation of degree eight in the uncontrolled setting (darker red, smaller) and in the controlled setting (lighter red, larger). The (approximation of) the "true" set (black) in the uncontrolled setting is represented as an ensemble of initial conditions randomly sampled within the stateconstraint set.

such as SeDuMi or SDPA. One possibility is parallelization; for instance, the free parallel solver SDPARA [43] allows for the approach to scale to larger dimensions. Alternatively, one can utilize one of the (few) commercial SDP solvers; in particular, the recently released MOSEK SDP solver seems to show far superior performance on our problem class, and therefore this may allow the approach to scale to larger dimensions. Finally, one can resort to customized structure-exploiting solutions; this is a promising direction of future research currently investigated by the authors. At this point it should be emphasized that, to the best of the authors' knowledge, all of the existing approaches providing approximations of similar quality experience similar or worse scalability properties.

Other directions of future research include the extension of the presented approach to inner approximations of MCI sets, to stochastic systems and to uncertain systems. Partial results on the inner approximations for the related problem of region of attraction computation already exist [26], albeit in uncontrolled setting only.

\section{REFERENCES}

[1] A. A. Ahmadi. Non-monotonic Lyapunov functions for stability of nonlinear and switched systems: theory and computation. Master's Thesis, MIT, Boston, 2008.

[2] L. Ambrosio. Transport equation and Cauchy problem for nonsmooth vector fields. In L. Ambrosio et al. (eds.), Calculus of variations and nonlinear partial differential equations. Lecture Notes in Mathematics, Vol. 1927, Springer-Verlag, Berlin, 2008.

[3] E. J. Anderson, P. Nash. Linear programming in infinite-dimensional spaces: theory and applications. Wiley, New York, 1987.

[4] J. P. Aubin, H. Frankowska. Set-valued analysis. Springer-Verlag, Berlin, 1990.

[5] M. A. Ben Sassi, A. Girard. Controller synthesis for robust invariance of polynomial dynamical systems using linear programming. System Control Letters 61(4):506-512, 2012.

[6] D. Bertsekas. Infinite time reachability of state-space regions by using feedback control. IEEE Trans. Autom. Control 17(5):604-613, 1972.

[7] A. G. Bhatt, V. S. Borkar. Occupation Measures for Controlled Markov Process: Characterization and Optimality. Annals of Probability, 24:1531-1562, 1996.

[8] F. Blanchini. Set invariance in control. Automatica, 35(11):17471767, 1999.

[9] F. Blanchini. Ultimate boundedness control for uncertain discrete time systems via set-induced Lyapunov functions. IEEE Trans. Autom. Control 39(2):428-433, 1994. 

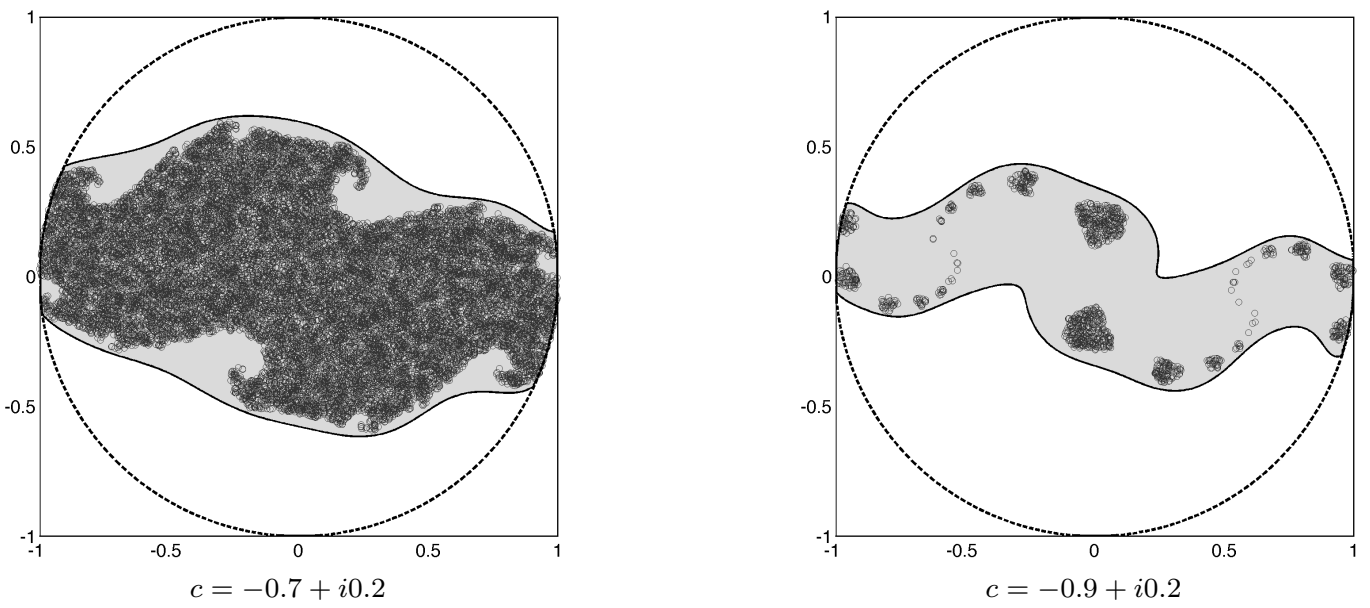

Fig. 1. Filled Julia set - polynomial outer approximation of degree 12 (light gray) and (an approximation of) the "true" set (dark grey) represented as an ensemble of initial conditions randomly sampled within the state-constraint set. The dashed line shows the boundary of the unit-ball state-constraint set.

[10] F. Blanchini, S. Miani, C. Savorgnan. Dynamic augmentation and complexity reduction of set-based constrained control. Proc. IFAC World Congress on Automatic Control, Seoul, South Korea, 2008.

[11] T. B. Blanco, M. Cannon, B. De Moor. On efficient computation of low-complexity controlled invariant sets for uncertain linear systems. Int. J. Control 83(7):1339-1346, 2010

[12] F. Blanchini, S. Miani. Set-theoretic methods in control. Birkhäuser, Boston, 2007.

[13] C. E. T. Dorea, J. C. Hennet. $(A, B)$-invariant polyhedral sets of linear discrete-time systems. J. Optim. Theory Appl., 103(3):521-542, 1999.

[14] G. Chesi. Domain of attraction; analysis and control via SOS programming. Lecture Notes in Control and Information Sciences, Vol. 415, Springer-Verlag, Berlin, 2011.

[15] V. Gaitsgory, M. Quincampoix. Linear programming approach to deterministic infinite horizon optimal control problems with discounting. SIAM J. on Control and Optimization, 48:2480-2512, 2009.

[16] E. G. Gilbert, K. T. Tan. Linear systems with state and control constraints: the theory and application of maximal output admissible sets. IEEE Trans. Autom. Control 36(9):1008-1020, 1991.

[17] R. Gondhalekar, J. Imura, K. Kashima. Controlled invariant feasibility - A general approach to enforcing strong feasibility in MPC applied to move-blocking. Automatica 45(12):2869-2875, 2009

[18] P. O. Gutman, M. Cwikel. An algorithm to find maximal state constraint sets for discrete-time linear dynamical systems with bounded controls and states. IEEE Trans. Autom. Control 32(3):251-254, 1987.

[19] D. Henrion, M. Korda. Convex computation of the region of attraction of polynomial control systems. arXiv:1208.1751, 2012.

[20] D. Henrion, J. B. Lasserre, J. Löfberg. Gloptipoly 3: moments, optimization and semidefinite programming. Optim. Methods and Software 24:761-779, 2009

[21] D. Henrion, J. B. Lasserre, C. Savorgnan. Approximate volume and integration for basic semialgebraic sets. SIAM Review 51:722-743, 2009.

[22] O. Hernández-Lerma, J. B. Lasserre. Discrete-time Markov control processes: basic optimality criteria. Springer-Verlag, Berlin, 1996.

[23] O. Kallenberg. Foundations of modern probability. Springer-Verlag, Berlin, 2010

[24] A. N. Kanatnikov, A. P. Krishchenko. Localization of compact invariant sets of discrete-time nonlinear systems. Int. J. Bifurcation and Chaos 21(7):2057-2065, 2011.

[25] E. C. Kerrigan. Robust constraint satisfaction: Invariant sets and predictive control. Ph.D. Thesis. Univ. Cambridge, UK, 2000.

[26] M. Korda, D. Henrion, C. N. Jones. Inner approximations of the region of attraction for polynomial dynamical systems. 9th IFAC Symposium on Nonlinear Control Systems (NOLCOS), Toulouse, 2013.

[27] A. P. Krishchenko. A. N. Kanatnikov. Maximal compact positively invariant sets of discrete-time nonlinear systems. Proc. IFAC World Congress on Automatic Control, Milano, Italy, 2011.

[28] T. G. Kurtz. Equivalence of stochastic equations and martingale problems. Stochastic Analysis 2010, 113-130, Springer-Verlag, Berlin, 2011
[29] A. N. Daryin, A. B. Kurzhanski. Parallel algorithm for calculating the invariant sets of high-dimensional linear systems under uncertainty. Computational Mathematics and Mathematical Physics 53(1):34-43, 2013.

[30] J. B. Lasserre. Moments, positive polynomials and their applications Imperial College Press, London, UK, 2009.

[31] M. Liu, S. Zhang, Z. Fan, M. Qiu. $\mathrm{H}_{\infty}$ State Estimation for DiscreteTime Chaotic Systems Based on a Unified Model. IEEE Trans. on Systems, Man, and Cybernetics - Part B, Cybernetics, 42(4):10531063, 2012.

[32] J. Löfberg. YALMIP : A toolbox for modeling and optimization in MATLAB. In Proc. IEEE CCA/ISIC/CACSD Conference, Taipei, Taiwan, 2004

[33] K. Margellos, J. Lygeros. Hamilton-Jacobi formulation for reachavoid differential games. IEEE Transactions on Automatic Control, 56:1849-1861, 2011.

[34] I. Mitchell, C. Tomlin. Overapproximating reachable sets by Hamilton-Jacobi projections. Journal of Scientific Computing, 19:323-346, 2003

[35] I. Pólik, T. Terlaky, Y. Zinchenko. SeDuMi: a package for conic optimization. IMA workshop on Optimization and Control, Univ. Minnesota, Minneapolis, 2007.

[36] M. Putinar. Positive polynomials on compact semi-algebraic sets. Indiana Univ. Mathematics Journal, 42:969-984, 1993.

[37] S. V. Raković. Parameterized robust control invariant sets for linear systems: theoretical advances and computational remarks. IEEE Trans. Autom. Control, 55(7):1599-1614, 2010.

[38] J. E. Rubio. Control and Optimization: The Linear Treatment of Nonlinear Problems. Manchester University Press, Manchester, UK, 1985

[39] R. Vidal, S. Schaert, J. Lygeros, S. Sastry. Controlled invariance of discrete time systems. HSCC, Lecture Notes on Computer Science, 1790, Springer-Verlag, Berlin, 2000.

[40] K. Starkov. Bounds for compact invariant sets of the system describing dynamics of the nuclear spin generator. Communications in Nonlinear Science and Numerical Simulation 14(6):2565-2570, 2009.

[41] K. Starkov. Estimation of the domain containing all compact invariant sets of the optically injected laser system. Int. J. Bifurcation and Chaos 17(11):4213-4217, 2007.

[42] M. Yamashita, K. Fujisawa, M. Fukuda, K. Kobayashi, K. Nakta, M. Nakata. Latest developments in the SDPA Family for solving large-scale SDPs. In M. Anjos, J. B. Lasserre (Eds.). Handbook on Semidefinite, Cone and Polynomial Optimization: Theory, Algorithms, Software and Applications. Springer, NY, USA, Chap. 24, 687-714, 2011.

[43] M. Yamashita, K. Fujisawa, M. Kojima. SDPARA: SemiDefinite Programming Algorithm paRAllel version. Parallel Computing 29:1053 1067, 2003.

[44] L. C. Young. Calculus of variations and optimal control theory. Sunders, Philadelphia, 1969.

[45] K. Wang, U. Vaidya. Transfer operator approach for computing domain of attraction. IEEE Conference on Decision and Control (CDC), Atlanta, GA, 2010. 